\title{
Perfil clínico epidemiológico de pacientes com perda auditiva
}

\section{Epidemiological clinical profile of patients with hearing loss}

\author{
Hudson Jose Cacau Barbosa ${ }^{1}\left(\mathbb{D}\right.$, Rafaela Arêas Aguiar ${ }^{1}\left(\mathbb{D}\right.$, Hugo Moura Campos Bernardes ${ }^{1} \mathbb{D}$, Romildo Rocha Azevedo Junior ${ }^{2}$ (D) \\ Débora Brizon Braga ${ }^{2}$ (D), Ana Rosa Murad Szpilman²
}

1. Discente do curso de Medicina pela Universidade Vila Velha (UVV), Vila Velha, ES, Brasil. 2. Docente do curso de Medicina pela Universidade Vila Velha (UVV), Vila Velha, ES, Brasil.

\section{Resumo}

Introdução: Perda Auditiva é uma deficiência relativamente comum, que afeta os indivíduos devido às várias causas, entre elas as adversidades dentro do processo laboral ou pelo próprio processo natural do envelhecimento. 0 diagnóstico tardio e o tratamento inadequado são fatores agravantes. Objetivo: traçar o perfil clínico-epidemiológico dos pacientes atendidos no ambulatório de Otorrinolaringologia da Policlínica da Universidade Vila Velha e obter a prevalência de perda auditiva, relacionando-a com as principais causas da ocorrência do déficit auditivo. Métodos: Foi realizado estudo observacional transversal, retrospectivo, por meio de consulta aos prontuários de 1275 pacientes, no período de abril de 2014 a abril de 2016 . Os dados foram analisados a partir do cálculo de frequência, absoluta e relativa, e aplicação do teste de qui-quadrado. Resultados: A faixa etária mais prevalente foi a dos idosos (38\%), que apresentaram Hipertensão Arterial Sistêmica e Diabetes Mellitus como principais comorbidades. As queixas principais foram hipoacusia (40,3\%) e zumbido (36,6\%). O diagnóstico relativo ao tipo de perda auditiva mais prevalente foi a Perda Auditiva Neurossensorial, seguida de Perda Auditiva Condutiva. 0 fator de risco mais prevalente foi exposição a ruídos (26\%). Conclusões: A perda auditiva associada ao zumbido foi a mais prevalente neste estudo. 0 completo diagnóstico audiológico associado a uma conduta adequada e precoce previnem complicações na saúde e no desenvolvimento pessoal.

Palavras-chave: Perfil de Saúde. Otolaringologia. Perda Auditiva. Serviços de Saúde.

\begin{abstract}
Introduction: Hearing Loss is a relatively usual disorder, which affects the individuals due to labor process adversities or due to the natural aging process itself. The late diagnosis and inadequate treatment are aggravating factors. Objective: to delineate the epidemiological clinical profile of patients attended in the otolaryngology ambulatory in Vila Velha University Policlinic and obtain the prevalence of hearing loss, relating it with the main causes of hearing deficit occurrence. Methods: A cross-sectional, retrospective observational study was carried out by consulting 1275 patients' medical records, from April 2014 to April 2016. Data were analyzed using absolute and relative frequency and chi-square test. Results: the most prevalent age range was the elderly (38\%), which presented Systemic Arterial Hypertension and Diabetes Mellitus as their main comorbidities. The main complains were hypoacusia (40,3\%) and buzzing $(36,6 \%)$. The most prevalent diagnosis was Sensorineural Hearing Loss followed by Conductive Hearing Loss. The most prevalent risk factor was noise exposure (26\%). Conclusions: The hearing loss associated to buzzing was the most prevalent in this study. The complete audiological diagnosis associated to early and proper conduct prevent health and personal development complications.
\end{abstract}

Key words: Health Profile. Otolaryngology. Hearing loss. Health services.

INTRODUÇÃO

A perda auditiva (PA) pode ser considerada uma das deficiências mais incapacitantes para o convívio do indivíduo em sociedade. Ela interfere na comunicação interpessoal, na linguagem e em várias outras atividades de aprendizagem do cotidiano. Isso acaba por gerar consequências psicológicas e de desenvolvimento às pessoas, uma vez que se verifica, nos adultos, que a PA se associa ao quadro de depressão, ao declínio cognitivo e à redução da capacidade funcional ${ }^{1}$. Já nos idosos, é comum ocorrer isolamento social e quadros depressivos, além da aceleração do declínio da cognição².

A avaliação audiológica tem como objetivo principal determinar a integridade do sistema auditivo, além de identificar tipo, grau e configuração da perda auditiva em cada orelha ${ }^{3,4}$.
Conceitualmente, a PA pode ser unilateral, bilateral, parcial ou total de 41 decibéis $(\mathrm{dB})$ ou mais, diagnosticada por audiometria nas frequências de 500,1000, $2000 \mathrm{~Hz}$. Tal diagnóstico determina o grau da perda auditiva, segundo a classificação de Lloyd e Kaplan, $\leq 25$ dB audição normal, 26 - 40 dB Perda auditiva de grau leve, $41-55 \mathrm{~dB}$ grau moderado, $56-70 \mathrm{~dB}$ grau moderadamente severo, $71-90 \mathrm{~dB}$ grau severo, $\geq 91 \mathrm{~dB}$ grau Profundo 4 .

Ao exame audiométrico é possível identificar os tipos de perda auditiva de acordo com a origem do defeito, classificando-a em perda auditiva condutiva, neurossensorial e mista. A perda auditiva condutiva (PAC) pode ser identificada quando há 
dificuldade da passagem de som entre o conduto auditivo externo, a membrana timpânica e os ossículos auditivos da orelha média. A perda auditiva neurossensorial (PANS) ocorre quando a cóclea ou a via de condução nervosa entre a orelha interna e o córtex cerebral está comprometida. Já a perda auditiva mista (PAM) é a associação dos dois tipos anteriores de $\mathrm{PA}^{5}$.

O tipo de Perda Auditiva segundo a audiometria, pode ser classificada: Perda Auditiva Condutiva (Limiares de via óssea menores ou iguais a $15 \mathrm{~dB}$ NA e limiares de via aérea maiores que 25 dB NA, com gap aéreo-ósseo maior ou igual a $15 \mathrm{~dB}$ ), Sensório-neural (Limiares de via óssea maiores do que $15 \mathrm{~dB}$ NA e limiares de via área maiores que 25 dB NA, com gap aéreoósseo de até $10 \mathrm{~dB}$ ), Mista (Limiares de via óssea maiores do que $15 \mathrm{~dB}$ NA e limiares de via aérea maiores que $25 \mathrm{~dB} N \mathrm{NA}$, com gap aéreo ósseo maior ou igual a $15 \mathrm{~dB})^{4}$.

A perda auditiva, considerada uma condição de etiologia variável, pode ser causada por fatores genéticos, ambientais, ou $a b_{0}{ }^{5}$. É importante notar que a prevalência da perda auditiva já é bastante significativa, atingindo cerca de 360 milhões de pessoas em todo o mundo. Essa alta prevalência mundial se deve principalmente ao aumento da expectativa de vida e da população de idosos, decorrentes da transição epidemiológica em muitos países ${ }^{6}$. De acordo com o Instituto Brasileiro de Geografia e Estatística (IBGE), essa condição está entre as principais deficiências presentes no país?

Os estudos realizados na população brasileira sobre esse assunto apresentam maior número de informações sobre os idosos, já que eles são os mais acometidos pela redução limiar da audição, ou Presbiacusia, devido ao próprio processo de envelhecimento ${ }^{2}$. A Presbiacusia, portanto, deve ser suspeitada quando existe perda auditiva bilateral, tipo neurossensorial e redução no limiar auditivo em ambas as orelhas, em geral, dos pacientes que apresentam cerca de 60 anos de idade ${ }^{8}$. Outras comorbidades associadas à perda auditiva são: a otite média, o diabetes, a hipertensão arterial sistêmica, o reumatismo, a depressão, o uso de medicamentos com grau de ototoxicidade, entre outros ${ }^{1}$.

Importante mencionar que a epidemiologia clínica é entendida como a aplicação ao indivíduo doente dos princípios e métodos da epidemiologia, ocupando-se especificamente da prática clínica por meio do estudo da variação e dos determinantes da evolução da doença, com foco em grupos de indivíduos doentes, com o doente como unidade de observação ${ }^{9}$.

Frisa-se que o levantamento do quantitativo de deficientes auditivos associando suas deficiências às respectivas causas de perda auditiva, bem como os fatores de risco, comorbidades, condições predisponentes e faixa etária de acometimento são informações que podem auxiliar no direcionamento de políticas e recursos para os serviços de saúde auditiva da população.

Tais informações podem ser incorporadas em programas já existentes na atenção básica de saúde (voltados para diabéticos, hipertensos, saúde do idoso, saúde mental, entre outros), possibilitando o diagnóstico precoce de PA adquirida na idade adulta, já que, grande parte dos programas de prevenção é geralmente direcionada à criança ${ }^{1}$.

Espera-se, com este estudo, conhecer a saúde auditiva da população estudada, além de possibilitar a intervenção precoce em pacientes com risco de PA. Este estudo servirá como base estatística e fonte de informação do perfil clínico-epidemiológico dos pacientes atendidos em um ambulatório de referência em diagnóstico audiológico em Vila Velha, Estado do Espírito Santo e pode servir como gatilho para novos estudos.

Assim sendo, objetivou-se traçar o perfil clínico-epidemiológico dos pacientes atendidos em um ambulatório de referência em diagnóstico audiológico em Vila Velha, Estado do Espírito Santo, e obter a prevalência de perda auditiva, verificando a associação da PA com as possíveis causas de ocorrência do déficit auditivo.

\section{MÉTODOS}

Foi realizado estudo observacional do tipo transversal, retrospectivo, por meio de consulta aos dados disponíveis nos prontuários dos pacientes que foram atendidos em um ambulatório de referência em diagnóstico audiológico em Vila Velha, Estado do Espírito Santo, no período de abril de 2014 a abril de 2016. Foram incluídos na pesquisa os prontuários de todos os pacientes atendidos pela primeira vez por esse serviço. Os prontuários de pacientes que não compareceram às consultas ou de pacientes que não tinham as informações preenchidas por completo foram excluídos do estudo.

O serviço de otorrinolaringologia em análise funciona como um programa interdisciplinar que envolve, além dos otorrinolaringologistas, outros profissionais da saúde como fonoaudiólogos, pediatras, neuropediatras e psicólogos.

Ao chegarem ao serviço, encaminhados via Unidade de Saúde, após avaliação médica, os pacientes foram atendidos pelos fonoaudiólogos que colheram uma anamnese inicial, e realizaram o exame de otoscopia. Em seguida, foram submetidos a exame audiométrico e posteriormente, encaminhados para os médicos otorrinolaringologistas que realizaram uma anamnese médica detalhada e exame clínico otorrinolaringológico completo, além de avaliarem a audiometria. A avaliação do exame audiométrico possibilitou fundamentar a conduta terapêutica adequada: tratamento clínico de afecções do ouvido (otite média supurada, otite serosa, otite média colesteatomatosa, sequela de otite crônica com perfuração de membra timpânica, indicação de uso do aparelho de amplificação sonora individual (AASI), solicitação de outros exames complementares, como Ressonância Magnética (RM), Tomografia Computadorizada (TC), Potencial Evocado Auditivo de Tronco Cerebral (PEATE), ou o encaminhamento para avaliação cirúrgica em outro serviço.

O perfil clínico e epidemiológico dos pacientes atendidos foi traçado com dados referentes ao sexo, idade, principal queixa 
(motivo) que levaram a buscar o serviço, o diagnóstico clínico junto a exames complementares, comorbidades, otoscopia e conduta do profissional diante do diagnóstico. Os diagnósticos foram categorizados de acordo com o capítulo VII da Classificação Estatística Internacional de Doenças e Problemas Relacionados à Saúde, Décima Revisão (CID-10). A idade foi categorizada por faixas etárias: crianças ( 0 a 14 anos), jovens (15 a 29 anos), adultos (30 a 59 anos) e idosos (60 anos ou mais) ${ }^{10}$.

Utilizou-se o programa Excel 7 para entrada dos dados e os resultados foram analisados por meio do programa SPSS $^{\circledR}$ for Windows, versão 20.0, por meio do teste do Qui-quadrado, a fim de se verificar a existência de associação entre o diagnóstico e a queixa principal, bem como com as outras variáveis levantadas para traçar o perfil epidemiológico. Para melhor esclarecer os resultados do qui-quadrado, foram também analisados os valores dos resíduos estandardizados e do coeficiente $\mathrm{V}$ de Cramer, quando o qui-quadrado acusou existência de associação, para também analisar, simultaneamente, o comportamento das variáveis e de categorias e a força da associação, uma vez que o teste de qui-quadrado apenas informa sobre a independência entre as variáveis, porém nada diz sobre o grau de associação existente. Existem, então, medidas específicas para atender a este fim, chamadas de medidas de associação, como o coeficiente Phi, o coeficiente de contingência (ou C de Pearson) e o coeficiente $V$ de Cramer. As medidas de associação normalmente variam entre 0 e 1 , isto é, desde ausência até a perfeita relação entre as variáveis. Os valores baixos indicam uma pequena associação, enquanto os elevados, uma grande associação ${ }^{11}$. Adotou-se nível de significância de $5 \%(p<0,05)$, como regra de decisão para os testes inferenciais.

Para a realização da pesquisa, solicitou-se a autorização da Coordenação do ambulatório de referência em diagnóstico audiológico em Vila Velha, Estado do Espírito Santo, e o estudo foi submetido e aprovado pelo Comitê de Ética em Pesquisa com Seres Humanos, sob número 1.559.143, em 10 de dezembro de 2015.

\section{RESULTADOS}

A faixa etária predominante dos indivíduos deste estudo foi igual ou superior a 60 anos, com uma amostra de 487 idosos, na maioria homens (41\%), seguida por indivíduos com idade entre 12 e 18 anos (35\%). A amostra foi constituída também por 303 adultos e 36 jovens, correspondendo a $24 \%$ e $3 \%$ da população estudada, respectivamente.

Verificou-se a relação da queixa principal com o diagnóstico. Para isso, considerou-se como queixa principal a alteração na fala, presença de otalgia e otorreia, hipoacusia, zumbido e outros. Como diagnóstico, definiram-se duas situações: Normal e Perda Auditiva (PA). Na Tabela 1, são apresentados os resultados dessa associação, de forma que foi verificada significância estatística $(p<0,05)$ entre diagnóstico e queixa principal.
Dos pacientes com diagnóstico de normalidade, 3,8\% ( $n=1)$ queixavam-se de alterações na fala, $11,5 \%(n=3)$ queixavam-se da presença de otalgia e otorreia e $19,2 \%(n=5)$ de hipoacusia. Daqueles diagnosticados com perda auditiva, 2,1\% ( $n=6)$ queixavam-se de alterações na fala, $0,7 \%(n=2)$ queixavam-se da presença de otalgia e otorreia, $44,1 \%(n=124)$ de hipoacusia e $42,3 \%(n=119)$ de zumbido. A hipoacusia foi a queixa mais frequente, presente em $40,3 \%(n=142)$ dos pacientes analisados, seguido de zumbido, que foi relatado por $36,6 \%$ $(n=129)$ pacientes. Mais da metade dos pacientes $(65,4 \%)$ que apresentaram diagnóstico de normalidade registraram outras queixas principais como tontura, cefaleia, atraso no desenvolvimento escolar, agressividade e plenitude auricular (Tabela 1).

Tabela 1. Definição de diagnóstico de acordo com a queixa principal.

\begin{tabular}{lrrrr}
\hline \multirow{2}{*}{ Queixa Principal } & \multicolumn{4}{c}{ Diagnóstico } \\
\cline { 2 - 5 } & Normal & PA $^{\mathrm{b}}$ & Outros $^{\mathrm{c}}$ & \multicolumn{1}{l}{ Total } \\
\hline Alteração fala & 3,8 & 2,1 & 2,2 & 2,3 \\
Otalgia/Otorreia & 11,5 & 0,7 & 11,1 & 2,8 \\
Hipoacusia & 19,2 & 44,1 & 28,9 & 40,3 \\
Zumbido & 0 & 42,3 & 22,2 & 36,6 \\
Outros $^{\mathrm{a}}$ & 65,4 & 10,7 & 35,6 & 17,9 \\
\hline Total & $\mathbf{1 0 0 , 0}$ & $\mathbf{1 0 0 , 0}$ & $\mathbf{1 0 0 , 0}$ & $\mathbf{1 0 0 , 0}$ \\
\hline
\end{tabular}

a OUTROS: Tontura, cefaleia, atraso no desenvolvimento escolar, agressividade e plenitude auricular.

b PA: Perda Auditiva

c OUTROS: Pacientes que ainda não fecharam o diagnóstico, sequelas neurológicas de hipóxia cerebral, percepção auditiva anormal, otite média, otite externa.

Frente à análise dos fatores de risco presentes na população, foi possível definir quatro diferentes diagnósticos: Normalidade, Perda Auditiva Condutiva (PAC), Perda Auditiva Neurossensorial (PANS) e Perda Auditiva Mista (PAM). Os pacientes que não receberam um diagnóstico fechado devido a sequelas neurológicas de hipóxia cerebral, perda auditiva anormal e presença de otite média e/ou externa, foram enquadrados no diagnóstico denominado "Outros", para facilitar a compreensão dos dados. O Gráfico 1 apresenta os principais fatores de risco encontrados na população e o diagnóstico dos indivíduos de acordo com esses critérios.

Dos 76 indivíduos que não apresentaram fatores de risco durante a anamnese, 15 foram diagnosticados com PANS e 7 com PAC. A otite de repetição foi o fator de risco encontrado em 21 pacientes, dos quais, 12 foram diagnosticados com PAC, 5 com PAM e 2 apresentaram normalidade. $O$ fator de risco mais prevalente foi a exposição a ruídos (26\%), encontrado em 64 indivíduos da amostra estudada. Destes, 49 (76,6\%) foram diagnosticados com PANS e o restante não teve um diagnóstico fechado. Outros fatores de risco como histórico familiar de PA e 
Gráfico 1. Diagnóstico dos indivíduos de acordo com os fatores de risco apresentados

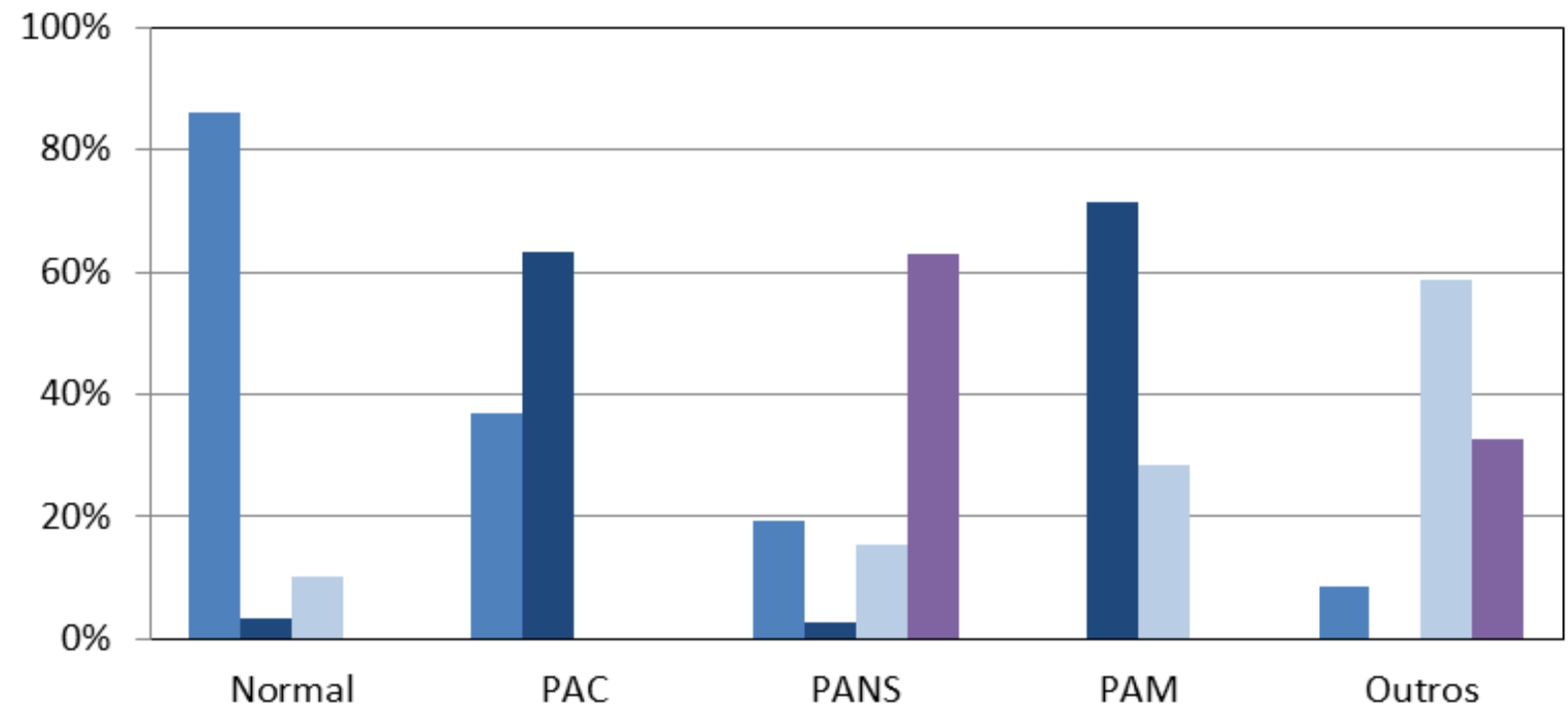

\section{Diagnóstico}

Ausente
Otite de repetição

\section{Outros}

Exposição à ruido histórico de permanência na UTI neonatal, foram encontrados em 85 pacientes, dos quais apenas 6 receberam diagnóstico de normalidade, sendo a maioria $(58,8 \%)$ diagnosticada com PANS (Gráfico 1).

A presença de comorbidades como distúrbios respiratórios (asma, rinite alergia, hipertrofia da adenoide), diabetes mellitus (DM) I e II e hipertensão arterial sistêmica (HAS) também foi analisada nesta amostra a fim de verificar sua relação com o diagnóstico determinado. A análise demonstrou significância estatística $(p<0,05)$ entre comorbidades e diagnóstico. Os resultados desta análise estão apresentados na Tabela 2.

Tabela 2. Diagnóstico dos pacientes de acordo com a presença de comorbidades.

\begin{tabular}{lrrrr}
\hline \multirow{2}{*}{ Comorbidades } & \multicolumn{4}{c}{ Diagnóstico } \\
\cline { 2 - 5 } & Normal & PA & Outros & Total \\
\hline Ausente & 58,5 & 32,9 & 22,7 & 34,8 \\
Distúrbios respiratórios & 2,4 & 2,9 & 13,6 & 4,3 \\
DM/HAS & 14,6 & 41,2 & 40,9 & 37,8 \\
Outros & 24,4 & 23 & 22,7 & 23,2 \\
\hline Total & $\mathbf{1 0 0 , 0}$ & $\mathbf{1 0 0 , 0}$ & $\mathbf{1 0 0 , 0}$ & $\mathbf{1 0 0 , 0}$ \\
\hline
\end{tabular}

a OUTROS: Otite Crônica, Anemia, Câncer de Próstata, Demência de Parkinson, TDAH, Síndrome de Down, Epilepsia, Doença do Refluxo Gastroesofágico.

A HAS e o DM foram as comorbidades mais frequentes nessa população $(37,8 \%)$, seguida de outras comorbidades não detalhadas $(23,2 \%)$. Os distúrbios respiratórios foram menos frequentes, acometendo apenas 4,3\% da população. Dos 243 pacientes diagnosticados com PA, 100 (41,2\%) apresentaram HAS e DM, o que indica forte influência dessas comorbidades com distúrbios auditivos. Distúrbios respiratórios também indicaram ter influência no acometimento do aparelho auditivo, já que metade dos pacientes com esses distúrbios foram diagnosticados com PA. Outras comorbidades como Otite Crônica, Anemia, Câncer de Próstata, Doença de Parkinson, Transtorno de Déficit de Atenção e Hiperatividade, Síndrome de Down, Epilepsia e Doença do Refluxo Gastroesofágico também mostraram forte influência no desenvolvimento da PA, já que, dos $76(23,2 \%)$ pacientes que apresentaram essas comorbidades, $56(73,6 \%)$ pacientes foram diagnosticados com PA (Tabela 2).

Foi verificada associação entre queixa principal e conduta, de forma que se verificou significância estatística $(p<0,05)$ entre esses fatores. A tabela 3 apresenta os resultados encontrados para essa associação. Como conduta, definiram-se os exames de Emissão Otoacústica, PEATE (Exame do Potencial Evocado Auditivo do Tronco Encefálico) e RCP (Reflexo Cócleo-Palpebral - usado principalmente em crianças).

De acordo com a análise, os exames mais indicados $(n=741)$ foram o "AIL Somente" e "AIL e Outros". A conduta "AIL Somente" e "AIL e Outros" foi definida principalmente para pacientes com queixa de hipoacusia $(40,8 \%)$, seguidos de pacientes com zumbido (35,2\%). A conduta "EBR Somente" e "EBR e Outros" foi mais indicada (51,8\%) para pacientes com queixas principais do tipo tontura, cefaleia e plenitude auricular, e para pacientes com queixas do tipo hipoacusia $(28,9 \%)$. Dos pacientes que receberam a conduta "EBR Somente" e "EBR e 
Outros" 2,4\% (n=2) apresentaram como queixa alteração na fala, $8,4 \%(n=7)$ otalgia e otorreia e $8,4 \%(n=7)$ zumbido. Dos que receberam a conduta "AIL Somente" e "AIL e Outros", 2,8\% $(n=21)$ apresentaram queixa de alteração na fala, 5,3\% ( $n=39)$ otalgia e otorreia e $15,9 \%(n=118)$ outras queixas principais (Tabela 3).

Tabela 3. Conduta realizada de acordo com a queixa principal.

\begin{tabular}{lrrr}
\hline & \multicolumn{3}{c}{ Conduta } \\
\cline { 2 - 4 } Queixa Principal & $\begin{array}{l}\text { EBRa somente/ } \\
\text { EBlLb somente/ }\end{array}$ & Total \\
& 2,4 & 2,8 & 2,8 \\
\hline Alteração fala & 8,4 & 5,3 & 5,6 \\
Otalgia e otorreia & 28,9 & 40,8 & 39,6 \\
Hipoacusia & 8,4 & 35,2 & 32,5 \\
Zumbido & 51,8 & 15,9 & 19,5 \\
Outros & $\mathbf{1 0 0 , 0}$ & $\mathbf{1 0 0 , 0}$ & $\mathbf{1 0 0 , 0}$ \\
\hline Total &
\end{tabular}

a EBR: Emissão Otoacústica + PEATE (Exame do Potencial Evocado Auditivo do Tronco Encefálico) + RCP (Reflexo Cócleo-Palpebral).

b AlL: Audiometria + Imitanciometria + Logoaudiometria.

c Outros: Exame de Ganho Funcional e Teste AASI com TPF (Aparelho de Amplificação Sonora Individual com Teste Padrão de Frequência).

\section{DISCUSSÃO}

O presente estudo teve maior prevalência de pacientes idosos (38\%), conforme encontrado em outros estudos epidemiológicos brasileiros ${ }^{12-14}$. Essa prevalência pode ser explicada devido ao aumento do número de idosos na população brasileira7, e pelo fato de serem os indivíduos mais propícios a desenvolver complicações na saúde em face do processo de envelhecimento, o que os leva a procurar mais os serviços de saúde do que os indivíduos de outras faixas etárias. A atenção aos pacientes idosos é de extrema importância já que problemas otorrinolaringológicos associados à depressão e à demência colaboram para o declínio social e a redução da qualidade de vida ${ }^{15}$.

A queixa principal mais frequente em todas as faixas etárias foi a de hipoacusia (40,3\%), conforme encontrado em estudos epidemiológicos semelhantes ${ }^{12,16}$, o que confirma a tendência da $\mathrm{OMS}^{9}$, de que a perda auditiva tem sido a deficiência sensorial mais frequente na população atual. Tendo em vista a importância da audição para o desenvolvimento pessoal e interação social, é necessário que haja maior atenção voltada para a saúde auditiva, não apenas para a qualidade de vida e saúde dos indivíduos, mas também pelo fato de que é mais vantajoso economicamente prevenir a surdez do que tratá-la com implante coclear e próteses ${ }^{17}$.

O zumbido foi a segunda queixa principal mais frequente, presente em $36,6 \%$ dos pacientes desse estudo. Esse resultado corrobora achados em outros estudos epidemiológicos semelhantes ${ }^{13,16}$. Tal sintoma faz que o indivíduo perceba um som em seus ouvidos ou em sua cabeça sem que haja qualquer fonte externa produzindo-o, o que causa grande incômodo ao paciente e impacto em sua qualidade de vida. É importante notar que, especialmente nos idosos, o zumbido é ainda mais impactante, reduzindo significativamente a atenção, a concentração e a alterando do sono, o que prejudica a realização de diferentes tarefas do cotidiano e pode colaborar para o aumento no risco de quedas ${ }^{16}$.

Dos pacientes que apresentaram alguma queixa, apenas $7,4 \%$ receberam diagnóstico de normalidade. Do total, $79,8 \%$ foram diagnosticados com perda auditiva e desses, a queixa mais frequente foi a hipoacusia $(44,1 \%)$, seguida de zumbido $(42,3 \%)$. A associação entre queixa e diagnóstico apresentou significância estatística $(p<0,05)$ e foi de baixa intensidade, de acordo com o V de Cramer ( $V=0,360)$, em consonância com outros estudos ${ }^{13,18}$.

Dos pacientes que apresentaram como fator de risco o histórico de PA familiar, 58,8\% foram diagnosticados com PANS. Esse achado correlaciona-se com o estudo que encontrou PANS em $64,4 \%$ dos participantes com esse mesmo histórico familiar19. De acordo com a literatura científica, a hereditariedade é uma das grandes responsáveis pelas perdas auditivas bilaterais, sendo a PANS a mais frequente quando há histórico familiar de $\mathrm{PA}^{20}$.

No presente estudo, o fator de risco mais prevalente foi a exposição a ruídos (26\%), em consonância com a literatura ${ }^{21}$. Dos pacientes com esse fator de risco, 76,6\% foram diagnosticados com PANS. A perda auditiva causada por exposição a ruído já é a principal causa de surdez nos Estados Unidos, de forma que o excesso de exposição a aparelhos de música portáteis tem causado com bastante frequência a PA temporária entre jovens e crianças ${ }^{22}$. Isso indica uma necessidade de orientação quanto ao uso desses aparelhos.

Há um consenso entre os pesquisadores de que a exposição ao ruído seja uma das frequentes causas de perda auditiva, estando associada ao zumbido, que se manifesta em 85$96 \%$ dos pacientes com perda auditiva ${ }^{8,23}$. Essa agressão ao sistema auditivo causada pela exposição ao ruído parece estar associada, também, a problemas cardiovasculares, alterações a curto prazo no sono dos indivíduos, disfunção da memória, além de problemas sociais como acidentes no trânsito ${ }^{24}$. Assim, devido aos efeitos biológicos e sociais dessa exposição, faz-se necessário maior atenção e assistência aos indivíduos mais vulneráveis.

Sabe-se, hoje, que o dano das células ciliadas provocado pelo ruído é desencadeado pelo estresse oxidativo nas células, culminando em lesão da cóclea e consequente formação de tecido cicatricial no Órgão de Corti em casos de perda auditiva permanente ${ }^{25}$. As drogas antioxidantes clinicamente importantes ajudam a prevenir danos causados por medicamentos como os aminoglicosídeos (neomicina, gentamicina), drogas quimioterápicas à base de platina (carboplatina, oxaliplatina) que são conhecidas por sua ototoxicidade ${ }^{25}$. Medidas não 
farmacológicas como programas de prevenção à exposição ao ruído ainda apresentam resultados não satisfatórios para prevenir danos ao aparelho auditivo ${ }^{23}$.

Com relação às comorbidades dos pacientes deste estudo, encontrou-se significância estatística $(p<0,05)$ de baixa intensidade $(V=0,199)$ entre comorbidades e diagnóstico. A análise indicou que as comorbidades mais prevalentes $(37,8 \%)$ foram a HAS e o DM e que destes, $80,6 \%(n=100)$ tiveram diagnóstico de PA, indicando que tais comorbidades exercem influência sobre o desempenho do aparelho auditivo. A prevalência de HAS e DM entre pacientes que procuraram o serviço de otorrinolaringologia também foi encontrada em outros estudos epidemiológicos ${ }^{12,13,16,18}$.

A literatura científica justifica a relação da HAS com a perda auditiva pelo fato de que, havendo comprometimento do sistema circulatório, o funcionamento da orelha interna também será comprometido, principalmente pela diminuição do fluxo sanguíneo capilar e do transporte de oxigênio ${ }^{26}$. Já a relação do DM com a perda auditiva se dá pelo fato de que ela é uma das comorbidades que mais colaboram para o descontrole da pressão arterial, sendo que a HAS está diretamente ligada à resistência insulínica. Além disso, a angiopatia, que é uma afecção comum no DM, interfere no suprimento sanguíneo da cóclea e pode levar à degeneração secundária do oitavo nervo craniano, conhecido como nervo auditivo ou acústico ${ }^{26}$.

Foi feita associação da queixa principal com a conduta estabelecida, de forma que a análise estatística encontrou significância $(p<0,05)$, de baixa intensidade $(V=0,291)$ nessa associação. Assim, quanto à conduta estabelecida, os exames Audiometria, Imitanciometria e Logoaudiometria foram os mais indicados $(90 \%)$, principalmente para os pacientes que registraram queixa de hipoacusia e zumbido. Tais exames também foram os mais indicados e realizados em outros estudos epidemiológicos, sendo enquadrados na avaliação audiológica clínica convencional ${ }^{14,27}$. A Imitanciometria, de acordo com alguns autores ${ }^{27,28}$, tende a ser menos frequente haja vista a dificuldade de obtenção do equipamento para sua realização. Embora seja um exame de extrema importância para avaliar a funcionalidade da membrana timpânica, os reflexos do estapédio (alterados quando em perdas auditivas), as condições da orelha média e para identificar se a lesão é coclear ou não, na maioria dos centros auditivos não é realizada ${ }^{28}$.

De forma geral, o diagnóstico mais prevalente para todas as faixas etárias deste estudo foi a PANS. Essa prevalência se assemelha ao encontrado em outros estudos ${ }^{12,13,29-31}$, sendo que a PANS tende a ser progressiva e a aumentar proporcionalmente com o avanço da idade ${ }^{32}$.

Ainda, de acordo com a análise estatística deste estudo, todas as associações realizadas apresentaram baixa intensidade, uma vez que o valor de $\mathrm{V}$ de Cramer para cada associação não se aproximara de 1 - quando se tem indicativo de forte associação. A baixa intensidade dessas associações pode ser justificada pelo fato de o estudo ser observacional, ou seja, sem um delineamento pré-definido, em que se controlam melhor as variáveis intervenientes, ficando, portanto, suscetível à influência de diversas variáveis externas.

\section{CONCLUSÃO}

A perda auditiva neurossensorial foi a mais prevalente neste estudo, e a hipoacusia e o zumbido foram as queixas mais frequentes entres os pacientes atendidos. Esses resultados demonstram a necessidade de maior atenção à saúde auditiva da população para todas as faixas etárias, tendo em vista que, uma audição saudável é de extrema importância para que o indivíduo possa desenvolver mecanismos de comunicação e interação social, indispensáveis para sua qualidade de vida.

Qualquer falha ou deficiência no sistema auditivo pode levar a grandes prejuízos na saúde e no bem-estar das pessoas, as quais são privadas de manter uma comunicação ampla e irrestrita e levadas ao isolamento social. Assim, o completo diagnóstico audiológico associado a uma conduta adequada e precoce previnem complicações na saúde e no desenvolvimento pessoal

\section{AGRADECIMENTOS}

Os autores expressam seu agradecimento ao apoio da Universidade Vila Velha (UVV). O estudo é fruto de pesquisa de Iniciação Científica da Universidade Vila Velha (UVV), em que os alunos receberam bolsa de Iniciação Científica como fonte de financiamento para a realização da pesquisa.

\section{REFERÊNCIAS}

1. Cruz, MS, Oliveira LR, Carandina L, Lima MCP, César CLG, Barros MBA, et al. Prevalência de deficiência auditiva referida e causas atribuídas: um estudo de base populacional. Cad Saúde Pública. 2009; 25 (5):1123-31. doi: http://dx.doi. org/10.1590/S0102-311X2009000500019.

2- Polanski JF. Presbiacusia. In: Piltcher OB, Costa SS, Maahs GS, Kuhl G. Rotinas em Otorrinolaringologia. Porto Alegre: Artmed; 2015. p. 83-86.

3- Lopes AC, Munhoz GS, Bozza A. Audiometria Tonal Limiar e de Altas Frequências. In: Boéchat EM, Menezes PL, Couto CM, Frizzo ACF, Scharlach RC, Anastasio ART. Tratado de Audiologia. Rio de Janeiro: Santos; 2015. p. 57-67.

4- Sistema de Conselhos Federal e Regionais de Fonoaudiologia. Manual de procedimentos em audiometria tonal limiar, logoaudiometria e medidas de imitância acústica. Brasília: Conselho Federal de Fonoaudiologia, 2013.

5. Melo US. Estudo epidemiológico e genético da surdez em dois municípios do estado da Paraíba, Brasil [dissertação]. São Paulo: Universidade de São Paulo; 2013.

6. Barros PMFB, Cavalcante TCF, Andrade AF. Audiologia em Comunidade: relato de experiência. Rev. CEFAC. Jul-Aug 2010; 12 (4): 626-32.

7. Instituto Brasileiro de Geografia e Estatística Censo Demográfico 2010[internet]. Rio de Janeiro: IBGE; 2011[acesso 2017 março 14]. Disponível em: http://www.ibge.gov.br/home/estatistica/populacao/censo2010/.

8. Sanchez TZ, Rocha SCM. Zumbido. In: Bento RF, Voegels RL, Sennes LU, Pinna FR, Jotz GP. Otorrinolaringologia Baseada em Sinais e Sintomas. São Paulo: 
Fundação Otorrinolaringologia; 2011. p. 39-43.

9. Barros H. Epidemiologia clínica: história e fundamentos para a sua compreensão. Rev. Port. Cir. 2013 Mar; (24): 51-56.

10. Organização Mundial da Saúde. WHO/PDH/97. 3. ed. Genova: WHO; 1997.

11. Pestana MH, Gageiro JN. Análise de Dados para Ciências Sociais: A Complementariedade do SPSS. 6. ed. Lisboa: Edições Sílabo Ltda; 2014.

12. Dimatos OC, Yudi Ikino CM, Philippi PA, Dimatos SP, Birck MC, Freitas PF. Perfil dos pacientes do programa de saúde auditiva do Estado de Santa Catarina atendidos no HU-UFSC. Arquivos Int. Otorhinolaryngol. 2011Jan-Mar; 15(1): 5966. doi: http://dx.doi.org/10.1590/S1809-48722011000100009.

13. Jardim DS, Macile FG, Lemos SMA. Perfil epidemiológico de uma população com deficiência auditiva. Rev CEFAC. 2016; 18(3): 746-57. doi: http://dx.doi. org/10.1590/1982-021620161833115.

14. Béria JU, Raymann BCW, Gigante LP, Figueiredo $A C L$, Jotz G, Roithman R, et al. Hearing impairment and socioeconomic factors: a population-based survey of an urban locality in southern Brazil. Rev Panam Salud Publica. 2007 Jun; 21(6): 381-7. PubMed PMID: 17761050.

15. Lavinsky J, Nudelmann AA. Perda Auditiva Induzida por Ruído. In: Piltcher OB, Costa SS, Maahs GS, Kuhl G. Rotinas em Otorrinolaringologia. Porto Alegre: Artmed; 2015. p. 86-90.

16. Ferreira LMBM, Ramos ANR Jr, Mendes EP. Caracterização do zumbido em idosos e de possíveis transtornos relacionados. Braz J Otorhinolaryngol. 2009; 75(2): 249-55. doi: http://dx.doi.org/10.1590/S0034-72992009000200015.

17. Cecatto SB, Garcia RID, Costa KS, Abdo TRT, Rezende CEB, Rapoport PB. Análise das principais etiologias de deficiência auditiva em Escola Especial "Anne Sullivan". Rev Bras Otorrinolaringol. 2003; 69(2): 235-40. doi: http:// dx.doi.org/10.1590/S0034-72992003000200014.

18. Gibrin PCD, Melo JJ, Marchiori LLM. Prevalência de queixa de zumbido e prováveis associações com perda auditiva, diabetes mellitus e hipertensão arterial em pessoas idosas. Rev CoDAS. 2013; 25(2): 176-80. doi: http://dx.doi. org/10.1590/S2317-17822013000200014.

19. Amorim AM, Carvalho CAF, Azevedo MF, Longhitano SB. Perda auditiva hereditária: relato de casos. Rev. CEFAC. 2008 Jan-Mar; 10(1): 117-25. doi: http://dx.doi.org/10.1590/S1516-18462008000100016.

20. Morales-Angulo C, Gallo-Terán J, Azuara N, Quintela JR. Etiology of severe/ profound, pre/perilingual bilateral hearing loss in Cantabria (Spain). Acta Otorrinolaringol Esp. 2004 Oct; 55(8): 351-5. PubMed PMID: 15552209.
21. Dias A, Cordeiro R, Corrente JE, Gonçalves CGO. Associação entre perda auditiva induzida por ruído e zumbidos. Cad. Saúde Pública. 2006 Jan; 22(1): 63-8. doi: http://dx.doi.org/10.1590/S0102-311X2006000100007.

22. Daniel E. Noise and Hearing Loss: A Review. J Sch Health. 2007 May; 77(5): 225-31. PubMed PMID: 17430434.

23. Sliwinska-Kowalska M, Davis A. Noise-induced hearing loss. Noise Health. 2012; 14(61): 274-80.

24. Basner M, Brink M, Birstow A, Kluizennar Y, Finegold L, Hong J, et al. ICBEN review of research on the biological effects of noise 2011-2014. Noise Health. 2015 Mar-Apr; 17(75): 57-82. doi: 10.4103/1463-1741.153373.

25. Silveira SCR. Ototoxicidade. In: Piltcher OB, Costa SS, Maahs GS, Kuhl G. Rotinas em Otorrinolaringologia. Porto Alegre, RS: Artmed; 2015. p. 90-96.

26. Fuess VLR, Cerchiari DP. Estudo da hipertensão arterial sistêmica e do diabetes mellitus como fatores agravantes da presbiacusia. Int Arch Otorhinolaryngol. 2003 Abr-Jun; 7(2): 116-21.

27 Zandavalli MB, Christmann LS, Garcez VRC. Rotina de procedimentos utilizados na seleção e adaptação de aparelhos de amplificação sonoro individual em centros auditivos na cidade de Porto Alegre, Brasil - RS. Rev CEFAC. 2009; 11(suppl 1): 106-15. doi: http://dx.doi.org/10.1590/S151618462009005000012

28. Russo ICP, Almeida K. Considerações sobre a seleção e adaptação de próteses auditivas para o idoso. In: Almeida K, lorio MCM. Próteses auditivas: fundamentos teóricos e aplicações clínicas. 2. ed. São Paulo: Lovise; 2003. p. 177-90.

29. Machado MS, Teixeira AR, Seimetz BM, Rosito LPS, Lopes FF, Costa SSD. Análise dos limiares auditivos de indivíduos atendidos em um ambulatório de otite média crônica. Clin Biomed Res. 2015; 35(Supl 1); 1-7.

30. Costi BB, Olchik MR, Gonçalves AK, Benin L, Fraga RD, Soares RS et al. Perda auditiva em idosos: relação entre autorrelato, diagnóstico audiológico e verificação da ocorrência de utilização de aparelhos de amplificação sonoro individual. Rev Kairós. 2014 Jun; 17(2): 179-92.

31. Martins SAB, Bassi I, Mancini PC. Perfil audiológico de idosos submetidos à reabilitação vestibular. Rev CEFAC. 2015; 17(3): 813-26. doi: http://dx.doi. org/10.1590/1982-0216201511714.

32. Meneses C, Mario MP, Marchori LDM, Melo JJ, Freitas ERFS. Prevalência de perda auditiva e fatores associados na população idosa de Londrina, Paraná: estudo preliminar. Rev CEFAC. 2010 Maio-Jun; 12(3): 384-92. doi: http://dx.doi. org/10.1590/S1516-18462010005000051.

\section{Como citar este artigo/How to cite this article:}

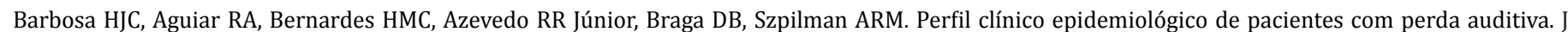
Health Biol Sci. 2018 Jul-Set; 6(4):424-430. 